(C) [2008] IEEE. Reprinted, with permission, from [Jun Ma, Guangquan Zhang, Team Situation Awareness Measurement Using Group Aggregation and Implication Operators, 2008, Proceedings of2008 3rd International Conference on Intelligent System and Knowledge Engineering]. This material is posted here with permission of the IEEE. Such ermission of the IEEE does not in any way imply IEEE endorsement of any of the University of Technology, Sydney's products or services. Internal or personal use of this material is permitted. However, permission to reprint/republish this material for advertising or promotional purposes or for creating new collective works for resale or redistribution must be obtained from the IEEE by writing to pubs-permissions@ieee.org. By choosing to view this document, you agree to all provisions of the copyright laws protecting it 


\title{
Team Situation Awareness Measurement Using Group Aggregation and Implication Operators
}

\author{
Jun Ma, Guangquan Zhang* \\ Faculty of Engineering and Information Technology, University of Technology, Sydney (UTS) \\ PO Box 123, Broadway, NSW 2007, Australia \\ E-mail: \{junm, zhangg\}@it.uts.edu.au
}

\begin{abstract}
Situation Awareness (SA) has been received much attention from human factors and ergonomics community during the past two decades. Team working plays a vital role in complex dynamic situations, increasing requirements for sharing perceptions and comprehension of a situation among team members and improving team situation awareness have been merged. This paper presents a team SA measurement (TSAM) method taking both qualitative and quantitative information into consideration. The TSAM method uses a hierarchy to depict team's shared mental model, applies linguistic terms to represent individual SA and adopts a group aggregation strategy to integrate individual SA through indicator-specified implication operators. Example has shown its effective.
\end{abstract}

\section{Introduction}

Situation Awareness (SA) is "the perception of the elements in the environment within a volume of time and space, the comprehension of their meaning and the projection of their status in the near future[5]". It is widely used in aircraft operator training. During the past two decades, it has been extended to complex dynamic systems where human factors are involved such as nuclear power plants [3], air traffic control [24, 12], and emergency response [2, 21]. Since team working plays an important role in organizational decision making in a dynamic situation, team situation awareness has drawn remarkable attentions of industrial and military communities recently.

Improving team SA becomes a crucial issue in complex dynamic systems, which is required to meet three main demands in those fields[6, 7, 20, 22, 13]. First, the increasing complexity of dynamic systems provides a great challenge

\footnotetext{
${ }^{*}$ Corresponding author
}

to decision makers or operators to recognize external environment timely and conduct ongoing analysis. Second, correct awareness about external environment is a basis for appropriate decision making. Particularly, team members often work simultaneously at different geographical locations. Research shows that team working can efficient reduce decision errors by individuals. Moreover, requirement for real world applications, such as developing friendly user interface for complex control systems and training operators in a complex dynamic environment, needs to be fulfilled. To improve team SA, an accurate measurement of team SA is important.

Since team SA is development with SA which focuses mainly on human factors analysis, traditional study of team SA is conducted through qualitative analysis methods. Qualitative techniques cannot be satisfactory to the aim of quantitative measurements for team SA. Thus quantitative techniques based on statistical models[19, 14, 23] and inference models[22] have been merged. However, these quantitative techniques have a common drawback, i.e., they completely replace qualitative information by numeric values. Accurate numeric values are easy to implement designed models at the cost of lacking meaningful qualitative information.

It is noticed that SA is information processing with a great amount of uncertainty. In the processing course, one applies background knowledge (forms the mental model of a situation) and uncertainty reasoning to handle perceptions with uncertainty (refers as situation models in [4]). An alternative technique for team SA measurement should combine qualitative and quantitative models. Research on linguistic decision making indicates that linguistic term is an efficient form to describe uncertain qualitative information $[9,8,25,27,16,18,17,11,10]$. Hence, in the light of research on linguistic information decisions, this paper presents a team SA measurement (TSAM) method by combining qualitative information process and quantitative computation. In the TSAM method, linguistic terms are used to describe individual and team SA. Moreover, the 
TSAM method applies group aggregation and implication operators to implement uncertain reasoning. The rest of the paper is organized as follows. In Section 2, an overview of the TSAM method is provided. Concrete steps of it are illustrated in Section 3. A case study on nuclear safeguards information management is discussed in Section 4 to illustrate the TSAM method. Finally, conclusions and future work are discussed in Section 5.

\section{Overview}

Developing team SA is an evolutionary process. First, team members generate individual SA based on personal mental models (i.e. relevant knowledge and experience) and individual situation models (i.e., perceptions of the external environment). Team members then share their individual SAs in the team through communication and cooperation. Next, the team forms a common awareness, which is treated as the team SA in this paper, about the situation. Finally, team's SA then is applied to reactions to the external environment to verify and modify. This is a repetitive course in a complex dynamic environment.

In order to measure team SA, two main factors are concerned in the TSAM method, i.e., the shared mental model of the team and the measurement of team members' awareness.

The shared mental model of a team is treated as the common accepted knowledge and experience about a given situation. In the TSAM method, the shared mental model is assumed to be a hierarchy with a set of identified indicators. Each node of the hierarchy is an identified indicator. An indicator at a lower level is called support an indicator at a higher level if there is a connection between them. An indicator is called an independent indicator if it is not supported by any indicator. Accordingly, an indicator is called a dependent indicator if it is supported by at least one indicator. Therefore, indicators in the mental model are divided into two groups.

A team member's individual SA is described as a covering on the identified indicators in the TSAM method. The covered indicator may be an independent one or a dependent one.

The shared mental model and individual SA are established based on the following consideration. Seen from viewpoint of uncertain information processing, the shared mental model plays the role of rule bases. The triggers of those rules are team member's perceptions of the external environment. That means team members' perception acts as the antecedent of uncertain reasoning. In general, one perception may trigger several rules. The TSAM method uses the triggered indicators of a perception to represent a perception. Moreover, team SA can be seen as the consequence of a series of uncertain reasoning. The TSAM method uses a hierarchy to describe the cause-and-effect between indicators. Therefore, a perception spreading from its covered indicators and stopping at the indicator to team SA implements the uncertainty reasoning. The team SA is thus formed.

Based on the division of indicators, the TSAM method includes two stages. At stage one, team SA on independent indicators is measured. At the second stage, team SA on dependent indicators are measured. A brief illustration of the TSAM is shown below.

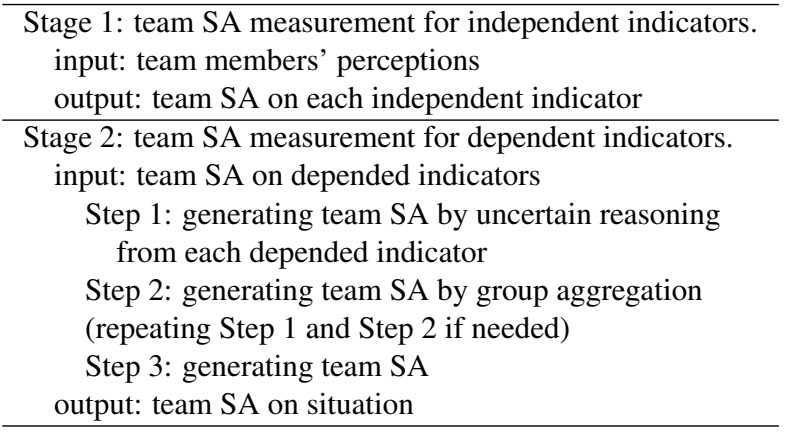

\section{Team SA Measurement}

Suppose a team $T=\left\{g_{1}, g_{2}, \cdots, g_{m}\right\}$ is working in a given environment to complete an assigned task. For each member $g_{i} \in T$, let $w_{i}(\in L W)$ be a linguistic weight attached to $g_{i}$ based on his/her professional background, experience, etc. Suppose a set IND of indicators for the team's mental model are identified and organized in a hierarchy. Let $\operatorname{ind}_{i j} \in$ IND be the $j$-th indicator at level $i$ to which a linguistic strength, denoted by $s_{i j}$, is assigned to it and $s_{i j} \in S$. Assume $\mathrm{IND}^{i}$ is the indicators covered by a perception of member $g_{i}, i=1,2, \ldots, m$.

\subsection{Team SA measurement for indepen- dent indicators}

The team SA measurement for independent indicators is implemented through aggregating individual SAs. In the following, let $S A_{i}$ be the individual SA of team member $g_{i}$.

For an independent indicator ind, let $G$ be the set of team members whose tasks cover indicator ind. Then team SA on indicator ind is obtained by

$$
\mathrm{tSA}=\operatorname{Agg}_{g \in G}\left(w_{g}, S A_{g}\right)
$$

where Agg is a selected aggregation operator[1, 26, 27]. Although $G$ may not include all team members in $T$, the result $t S A$ can still be taken as the team SA under the assumption that team members completely trust others in the team because this is a fundamental basis for team collaboration. 


\subsection{Team SA measurement for dependent indicators}

The main work at the second stage is to obtain team SA on dependent indicators through integrating team SA on the indicators it depends on.

Suppose ind is a dependent indicator which depends on indicators $\operatorname{ind}_{1}, \operatorname{ind}_{2}, \ldots, \operatorname{ind}_{n}$. Assume that $\mathrm{tSA}_{i}$ is the team SA on indicator ind ${ }_{i}$. Then, team SA on indicator ind is obtained by the following steps.

Step 1: generate team SA on ind through each depended indicator $\operatorname{ind}_{i}$.

Dependent relationship between ind and ind ${ }_{i}$ describes the internal cause-and-effect relationship which can be formally illustrated by

$$
P\left(\text { ind }_{i}\right) \rightarrow P(\text { ind }),
$$

that is to say, when some fact about indicator ind i $_{i}$ occurs, a certain fact about indicator ind occurs then. Furthermore, this relationship can also be formalized by

$$
P(\text { ind })=P\left(\text { ind }_{i}\right) \circ\left(P\left(\text { ind }_{i}\right) \rightarrow P(\text { ind })\right),
$$

where o depicts the logical connective "AND". To determine the operators "०" and " $\rightarrow$ ", we first select an implication operator as " $\rightarrow$ ", and then determine the "AND" for "o". For example, if the used implication operator is the Łukasiewicz implication operator $a \rightarrow b=\min \{1,1-a+$ $b\}$ where $a, b \in[0,1]$, then the operator $\circ$ is defined by

$$
a \circ b=\min \{a, b\}, \quad a, b \in[0,1] .
$$

However, in real situation, we need not directly use the implication operator because $P\left(\right.$ ind $\left._{i}\right) \rightarrow P($ ind $)$ and $P\left(\right.$ ind $\left._{i}\right)$ can be replaced by the strength $s_{i}$ and the $\mathrm{tSA}_{i}$, respectively. The thing only needs to do is determining the "AND" by the implication operator and the smallest truth-value in a non-classical logic for uncertainty reasoning where the implication operator is applied.

Therefore, team SA on indicator ind from indicator ind $\mathrm{i}_{i}$ is generated by under an implication operator " $\rightarrow$ "

$$
\overline{\mathrm{tSA}}_{i}=\mathrm{tSA}_{i} \circ s_{i} \text {. }
$$

Step 2: generate team SA on indicator ind by group aggregation for indicator groups.

Group aggregation is a strategy discussed by Liu et al. [15] for nuclear safeguards information management. The basic idea of it is dividing indicators into several groups according to their strengths and selecting strength specific aggregation operators for different groups. The TSAM method applies the group aggregation. Suppose indicators ind $_{1}, \operatorname{ind}_{2}, \ldots$, ind $_{n}$ are divided into $p$ groups $I N D^{(k)}$, $k=1,2, \ldots, p$. For group $I N D^{(k)}$, let $\operatorname{Agg}^{(k)}$ be a selected aggregation operator, then the team SA on $I N D^{(k)}$ is generated by

$$
\mathrm{tSA}^{(k)}=\operatorname{Agg}^{(k)}\left(s^{(k)} ;\left\{{\overline{\mathrm{tSA}_{j}}}_{j} \mid \operatorname{ind}_{j} \in I N D^{(k)}\right\}\right),
$$

where $s^{(k)}$ is the strength of indicators in that group. The $\mathrm{tSA}^{(k)}$ is thus the team awareness on ind through $I N D^{(k)}$.

Step 3: generate team SA on indicator ind by aggregating group team $\mathrm{SA} \mathrm{tSA}^{(k)}$.

Suppose the obtained team SA for $p$ groups are $\mathrm{tSA}^{(k)}$, $k=1,2, \ldots, p$ and Agg is a selected aggregation operator for indicator ind, then the team SA on ind is generated by

$$
\mathrm{tSA}_{i n d}=\operatorname{Agg}\left(\left(\mathrm{tSA}^{(1)}, s^{(1)}\right), \cdots,\left(\mathrm{tSA}^{(p)}, s^{(p)}\right)\right) .
$$

By above three steps, team SA on a dependent indicator ind is generated. Repeating the process in Stage 2, the team SA about the given situation is measured.

\section{Case Study}

\subsection{Case illustration and settings}

In this section, we apply the TSAM method to an example[15].

Suppose a team of four members, $E_{1}, E_{2}, E_{3}, E_{4}$, is assigned a task to inspect whether an organization is conducting a kind of nuclear activity. The team's mental model about the nuclear activity is shown in Figure 1. Team members report their observations about the organization's nuclear activity independently. Table 1 is the report of their observations where the observations are labels for terms taken from a linguistic term set $S=\left\{s_{0}=\right.$ impossible, $s_{1}=$ almost impossible, $s_{2}=$ slightly possible, $s_{3}=$ quite possible, $s_{4}=$ possible, $s_{5}=$ high possible, $s_{6}=$ absolutely possible $\}$. Let weight of each member be $E_{1}=$ $r_{3}, E_{2}=r_{5}, E_{3}=r_{4}$, and $E_{4}=r_{2}$, where $r_{i}$ in $W=\left\{r_{0}=\right.$ none, $r_{1}=$ very low, $r_{2}=$ low, $r_{3}=$ medium, $r_{4}=$ high, $r_{5}=$ very high, $r_{6}=$ perfect $\}$.

For convenience, we use the fuzzy numbers in Figure 2 and Figure 3 to describe these linguistic terms. Notice that "weak" is near $s_{1}$, "medium" is near $s_{2}$, "strong" is near $s_{6}$ by the distance defined in [28], we also use $s_{1}, s_{2}$ and $s_{6}$ to replace them respectively.

\subsection{Case solution}

Based on the above assumptions, we apply the TSAM method as follows.

First, the team SA measurement is obtained for independent indicators. Taking indicator "gaseous diffusion barriers (id 266)" for instance, members $E_{2}, E_{3}$, and $E_{4}$ has 


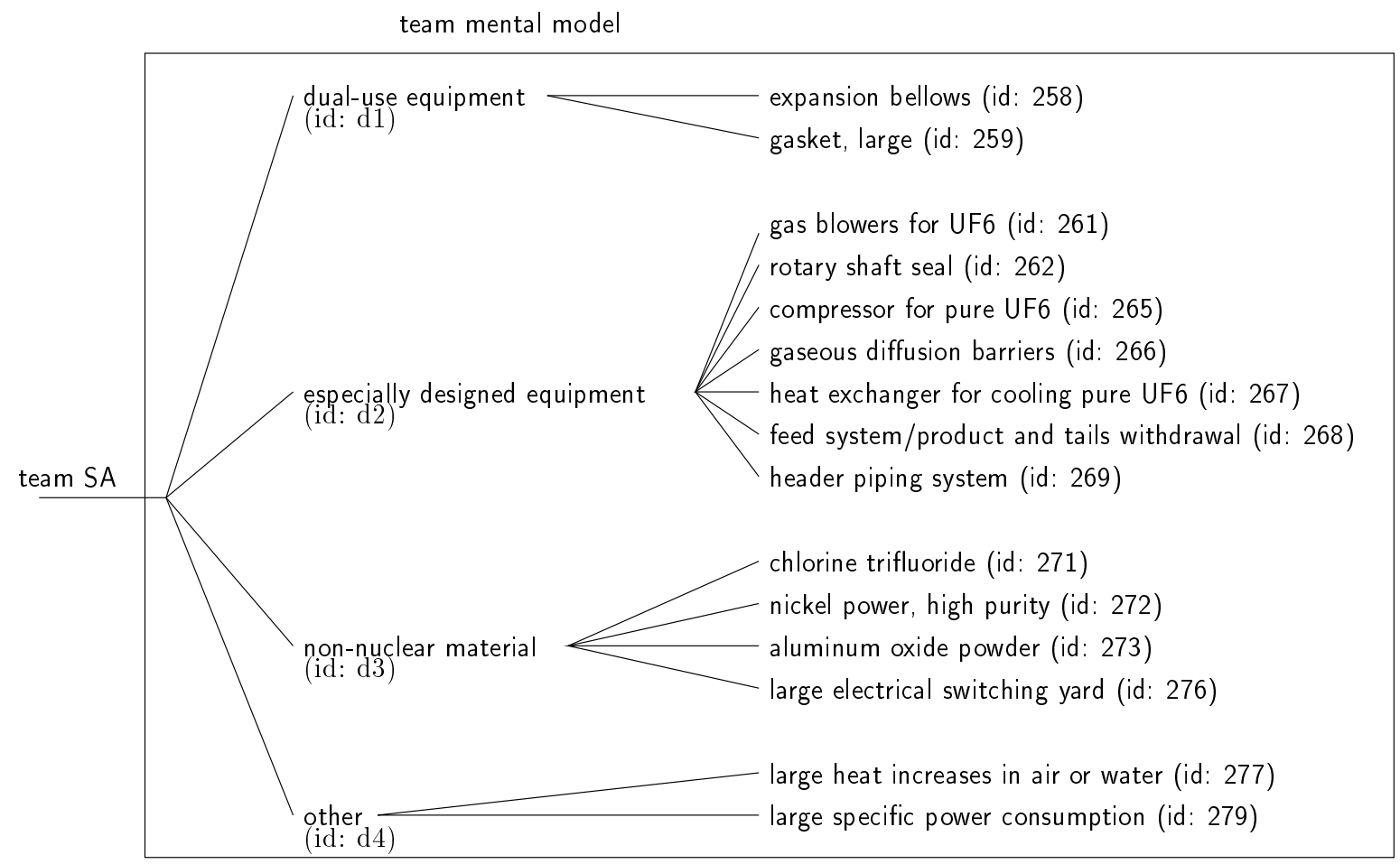

Figure 1. Team's mental model about a kind of nuclear activity.

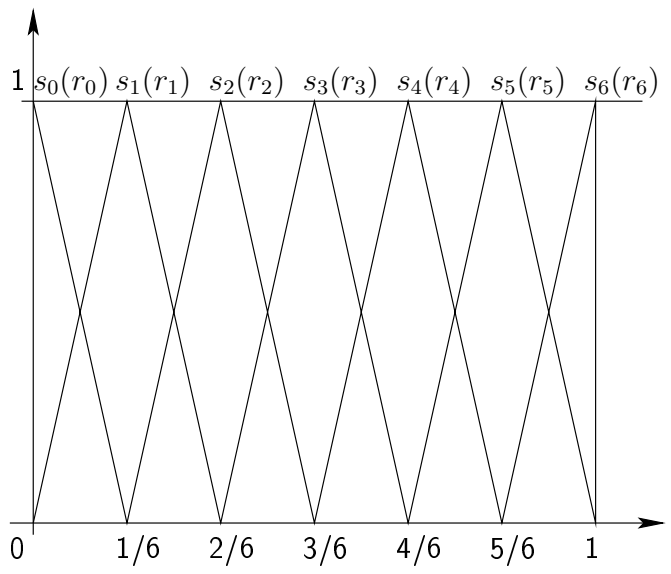

Figure 2. Fuzzy numbers used for linguistic terms.

reported observations for this indicator. Hence, these observations are aggregated. Here, we apply the fuzzy method in [28] to the aggregation, i.e.

$$
t S A_{266}=s_{5} \otimes r_{5} \oplus s_{4} \otimes r_{4} \oplus s_{6} \otimes r_{2} \approx s_{4} .
$$

Thus $s_{4}$ is taken as the awareness of the team on this indicator. Similarly for other independent indicators, the team's SA are calculated and list in Table 2.

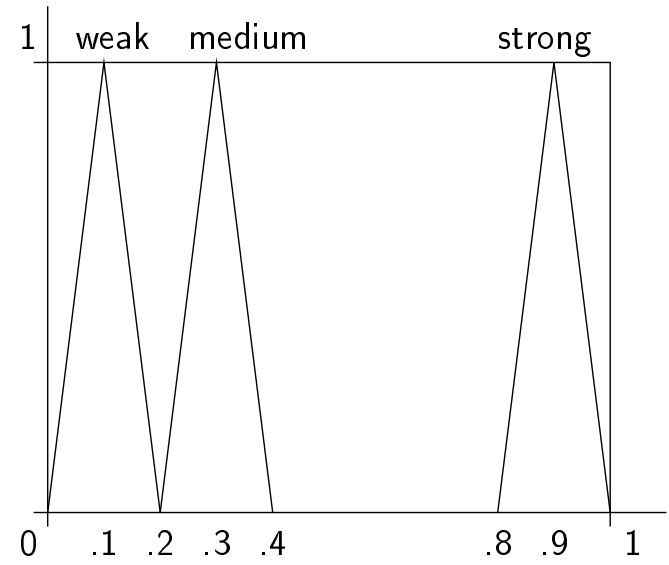

Figure 3. Fuzzy numbers used for indicator strengths.

Secondly, the team SA is obtained for dependent indicators. Taking indicator "especially designed equipment" (ind with id 2) for example, this indicator depends on seven indicators. Hence contribution to team SA on ind from each independent indicator is first calculated. For example, to obtain the contribution from indicator "gas blowers for UF6," the TSAM method selects the min operator Eq. (5). Then 
Table 1. Reported observations.[15]

\begin{tabular}{|c|c|c|c|c|c|}
\hline \multicolumn{4}{|c|}{ Observations } & Indicator IDs & Strength \\
\hline$E_{1}$ & $E_{2}$ & $E_{3}$ & $E_{4}$ & & \\
\hline 6 & 6 & 6 & 5 & 258 & weak \\
\hline 2 & - & 5 & - & 259 & weak \\
\hline- & 2 & 3 & 6 & 261 & medium \\
\hline 4 & 3 & - & - & 262 & medium \\
\hline 4 & - & 4 & - & 265 & strong \\
\hline- & 5 & 4 & 6 & 266 & strong \\
\hline 5 & 3 & 6 & - & 267 & strong \\
\hline 1 & - & 2 & 4 & 268 & weak \\
\hline 5 & - & 6 & 4 & 269 & weak \\
\hline 3 & 2 & - & 4 & 271 & medium \\
\hline- & 2 & 3 & - & 272 & medium \\
\hline- & 2 & 2 & 3 & 273 & weak \\
\hline 3 & - & 5 & 5 & 276 & weak \\
\hline 6 & 3 & - & - & 277 & weak \\
\hline - & 3 & 5 & 6 & 279 & weak \\
\hline
\end{tabular}

Table 2. Team's SA for independent indicators.

\begin{tabular}{l|cccccccc}
\hline Ind ID & 258 & 259 & 261 & 262 & 265 & 266 & 267 & 268 \\
\hline$t S A$ & 5 & 3 & 3 & 4 & 3 & 4 & 4 & 2 \\
\hline \hline Ind ID & 269 & 271 & 272 & 273 & 276 & 277 & 279 & \\
\hline$t S A$ & 4 & 2 & 2 & 2 & 3 & 3 & 3 & \\
\hline \hline
\end{tabular}

$\overline{\mathrm{tSA}}_{261}$ is

$$
\overline{\mathrm{tSA}}_{261}=\min \left\{s_{3}, w_{\text {medium }}\right\} \approx s_{2} .
$$

Similarly, the contributions of other independent indicators are obtained as below.

\begin{tabular}{lccccccc}
\hline Ind ID & 261 & 262 & 265 & 266 & 267 & 268 & 269 \\
\hline$\overline{\mathrm{tSA}}$ & 2 & 2 & 3 & 4 & 4 & 1 & 1 \\
\hline
\end{tabular}

Following above step, the TSAM method applies group aggregation to these seven indicators. As indicator 261 and 262 have same strength, they are aggregated by

$$
\mathrm{tSA}_{261,262}=\min \left\{s_{2}, s_{2}\right\}=s_{2} .
$$

Similarly, the TSAM method applies the max operator to indicator $265--267$ because their strength is strong and obtains

$$
\mathrm{tSA}_{265-267}=\max \left\{s_{3}, s_{4}, s_{4}\right\}=s_{4} .
$$

For indicators 268 and 269, the TSAM method uses the min operator and gets

$$
\mathrm{tSA}_{268,269}=s_{2} \text {. }
$$

Finishing the group aggregation, the TSAM method applies the technique in [28] to get team SA on "gas blowers for UF6" as

$$
\mathrm{tSA}_{\text {ind }}=s_{2} \otimes s_{2} \oplus s_{4} \otimes s_{6} \oplus s_{1} \otimes s_{1} \approx=s_{3} .
$$

Hence the team SA is "slightly possible."

Similarly for other dependent indicators, the team SAs are obtained:

$$
\mathrm{tSA}_{1}=s_{1} \quad \mathrm{tSA}_{3}=s_{2} \quad \mathrm{tSA}_{4}=s_{1} .
$$

Finally, based on the team SA on indicators d1, d2, d3, and $\mathrm{d} 4$, an overall team awareness on the situation can be obtained. Suppose strengths for indicators d1, d2, d3, and d4 are $s_{2}, s_{6}, s_{2}$, and $s_{1}$, then the overall team SA is near $s_{2}$. This indicates that the organization has slight possibility to conduct the very kind of nuclear activity.

\section{Conclusions and Future Works}

Efficiently measuring a team's awareness for a situation is a vital issue in complex dynamic management. This paper proposed a TSAM method to measure team SA which takes both qualitative information reasoning and quantitative information computation into consideration and uses indicator-specified implication operators and aggregation operators to generate team SA from individual SA. The TSAM method has been implemented in decision software. More presentation and discussion of this software will be in our following papers.

Because the core feature of developing team SA is the communication and collaboration among members, a detailed analysis of the specific nature of team communication and cooperation may contribute to establish an effective approach for developing team SA and supporting appropriate decision, which is exactly our future work.

\section{Acknowledgements}

The work presented in this paper was supported by Australia Research Council (ARC) under discovery grants DP0559213 and DP0880739.

The authors sincerely appreciate the advice and suggestions of professor Da Ruan from Belgian Nuclear Research Center (SCK·CEN), Belgium.

\section{References}

[1] G. Beliakov and J. Warren. Appropriate choice of aggregation operators in fuzzy decision support systems. IEEE Transactions on Fuzzy Systems, 9(6):773-784, 2001. 
[2] A. Blandford and B. L. W. Wong. Situation awareness in emergency medical dispatch. International Journal of Human-Computer Studies, 61:421-452, 2004.

[3] P. V. Carvalho, I. L. dos Santos, and M. C. Vidal. Safety implications of cultural and cognitive issues in nuclear power plant operation. Applied Ergonomics, 37:211-223, 2006.

[4] N. J. Cooke, R. J. Stout, and E. Salas. A knowledge elicitation approach to the measurement of team situation awareness. In M. McNeese, M. R. Endsley, and E. Salas, editors, New Trends in Cooperative Activities: System Dynamics in Complex Settings, pages 114-139. Human Factors, Santa Monica, CA, 2001.

[5] M. R. Endsley. Design and evaluation for situation awareness enhancement. In Proceedings of the Human Factors Society 32nd Annual Meeting, pages 97-101, Santa Monica, CA, 1988. Human Factors Society.

[6] M. R. Endsley. Toward a theory of situation awareness in dynamic systems: Situation awareness. Human Factors, 37(1):32-64, 1995.

[7] M. R. Endsley. Measurement of situation awareness in dynamic systems. Human Factors, 37(1):65-84, 1995 b.

[8] F. Herrera, E. Lopez, and M. Rodriguez. A linguistic decision model for promotion mix management solved with genetic algorithms. Fuzzy Sets and Systems, 131:47-61, 2002.

[9] E. Herrera-Viedma, F. Herrera, L. Martínez, J. C. Herrera, and A. G. López. Incorporating filtering techniques in a fuzzy linguistic multi-agent model for information gathering on the web. Fuzzy Sets and Systems, 148:61-83, 2004.

[10] N. Ho and H. V. Nam. An algebraic approach to linguistic hedges in zadeh's fuzzy logic. Fuzzy Sets and Systems, 129:229-254, 2002.

[11] N. Ho and W. Wechler. Hedge algebras: an algebraic approach to structure of sets of linguistic truth values. Fuzzy Sets and Systems, 35:281-293, 1990.

[12] D. B. Kaber, C. M. Perry, N. Segall, C. K. McClernon, and L. J. P. III. Situation awareness implications of adaptive automation for information processing in an air traffic controlrelated task. International Journal of Industrial Ergonomics, 36(5):447-462, 2006.

[13] T. Kanno, K. Nakata, and K. Furuta. A method for conflict detection based on team intention inference. Interacting with Computers, 18(4):1-23, 2006.

[14] A. Kirlik and R. Strauss. Situation awareness as judgment i: Statistical modeling and quantitative measurement. International Journal of Industrial Ergonomics, 36:463-474, 2006.

[15] J. Liu, D. Ruan, and R. Carchon. Synthesis and evaluation analysis of the indicator information in nuclear safeguards applications by computing with words. Int. J. Appl. Math. Comput. Sci., 12(3):449-462, 2002.

[16] J. Lu, G. Zhang, and D. Ruan. Intelligent multi-criteria fuzzy group decision-making for situation assessments. Soft Computing, 12(3):289-299, 2008.

[17] J. Lu, G. Zhang, D. Ruan, and F. Wu. Multi-Objective Group Decision Making - Methods, Software and Applications with Fuzzy Set Technology. Imperial College Press, London, 2007.
[18] J. Ma, D. Ruan, Y. Xu, and G. Zhang. A fuzzy-set approach to treat determinacy and consistency of linguistic terms in multi-criteria decision making. International Journal of Approximate Reasoning, 44:165-181, 2007.

[19] J. S. McMarley, C. D. Wickens, J. Goh, and W. J. Horrey. A computational model of attention/situation awareness. In Proceedings of the 46th Annual Meeting of the Human Factors and Ergonomic Society, Santa Monica, 2002. Human Factors and Ergonomics Society.

[20] P. Salmon, N. Stanton, G. Walker, and D. Green. Situation awareness measurement: a review of applicability for $\mathrm{c} 4 \mathrm{i}$ environments. Applied Ergonomics, 37:225-238, 2006.

[21] A. M. Schaafstal, J. H. Johnston, and R. L. Oser. Training teams for emergency management. Computers in Human Behavior, 17:615-626, 2001.

[22] Y. Shu and K. Furuta. An inference method of team situation awareness based on mutal awareness. Cognition Technology \& Work, 7:272-287, 2005.

[23] R. Strauss and A. Kirlik. Situation awareness as judgment II: Experimental demonstration. International Journal of Industrial Ergonomics, 36:475-484, 2006.

[24] J. Uhlarik and D. A. Comerford. A review of situation awareness literature relevant to pilot surveillance functions. Technical Report DOT/FAA/AM-02/3, Department of Psychology, Kansas State University, Manhattan, KS, March 2002.

[25] R. Yager and M. Detyniecki. Ranking fuzzy numbers using $\alpha$-weighted valuations. International Journal of Uncertainty, Fuzziness and Knowledge Based Systems, 8:573-591, 2000.

[26] R. R. Yager. Families of OWA operators. Fuzzy Sets and Systems, 59:125-148, 1993.

[27] R. R. Yager. OWA aggregation over a continuous interval argument with applications to decision making. IEEE Transactions on Systems, Man, and Cybernetics-Part B: Cybernetics, 34(5):1952-1963, 2004.

[28] G. Zhang and J. Lu. Information integration based team situation assessment in an uncertain environment. In D. Ruan, P. D'hondt, P. F. Fantoni, M. D. Cock, M. Nachtegael, and E. E. Kerre, editors, Applied Artificial Intelligence, pages 441-448, Genoa, Italy, August 2006. World Scientific Press. 